\title{
Expression of CD44s in Incidental Prostate Cancer Is More Strongly Associated with Gleason Scores on Subsequent Radical Prostatectomies than Conventional Prognostic Parameters
}

\author{
Sven Gunia ${ }^{a}$ Matthias May ${ }^{b}$ Stefan Koch ${ }^{a}$ Manfred Dietel ${ }^{c}$ \\ Andreas Erbersdobler ${ }^{\mathrm{C}}$ \\ a Department of Pathology, HELIOS Clinic Bad Saarow, Charité-University Medicine Teaching Hospital,

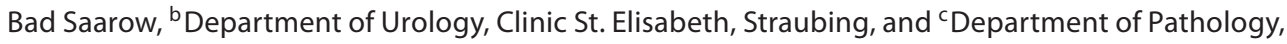 \\ Campus Charité Mitte, Charité-University Medicine Berlin, Berlin, Germany
}

\section{Key Words}

Incidental prostate cancer $\cdot$ Radical prostatectomy ·

Gleason scores $\cdot$ Immunohistochemistry $\cdot$ CD44s, standard isoform

\begin{abstract}
Objective: Incidental prostate cancer (IPC) has a substantially variable clinical course which cannot be predicted by conventional histopathologic examination of transurethral resection specimens of the prostate (TURP chips). Therefore, efforts should be directed towards defining the natural history of individual IPC. Recently, expression of CD44s (standard isoform), a transmembranous glycoprotein, has been linked to prognostic outcome in prostate cancer, but its prognostic role in IPC has been neglected so far. Methods: We present a multicentre study which evaluates immunohistochemically the largest cohort of IPC patients to date, aiming to correlate CD44s expression in the TURP chips with histopathologic outcome parameters (Gleason scores and histologic staging) performed on subsequent radical prostatectomies (RPs) in a cohort of 54 patients who underwent prostatectomy due to IPC. Results: CD44s expression recorded in the TURP chips showed a stronger (inverse) association with Gleason scores performed on the correspond-
\end{abstract}

ing RPs than did other conventional prognostic variables and, therefore, might become a valuable adjunct to better predict outcome in IPC prior to radical prostatectomy. Conclusion: Advanced prospective studies should aim to define cut-point values of CD44s expression for separating aggressive tumours from their indolent counterparts, and should also assess possible associations with clinical follow-up data (e.g. progression-free survival) in IPC.

Copyright $\odot 2009$ S. Karger AG, Basel

\section{Introduction}

Incidental prostate cancer (IPC) is defined as a clinically unapparent tumour discovered after histologic examination of the specimen obtained by open prostatectomy or transurethral resection of the prostate (TURP) [1]. Its overall incidence in TURPs performed for clinically benign prostatic hypertrophy varies between 13 and $22 \%$ [2]. The currently established subdivision of the disease into 2 histopathologic stages (T1a and T1b) according to the latest TNM classification system has gained wide acceptance, with those working in the field having become familiar with it [3].

\section{KARGER}

Fax +4161306 1234 E-Mail karger@karger.ch www.karger.com
(C) 2009 S. Karger AG, Basel

$1015-2008 / 09 / 0766-0286 \$ 26.00 / 0$

Accessible online at:

www.karger.com/pat
Sven Gunia, MD

Department of Pathology, HELIOS Clinic Bad Saarow

Charité-University Medicine Teaching Hospital, Pieskower Strasse 33

DE-15526 Bad Saarow (Germany)

Tel. +49 336317 2312, Fax +49 336317 3010, E-Mail sven.gunia@helios-kliniken.de 
However, owing to its substantially variable natural history, clinical management ('wait and watch' vs. radical surgery) remains challenging since the individual clinical course of IPC cannot be predicted by means of conventional histopathologic examination of TURP chips. In particular, there is often disagreement between the Gleason score on TURP and prostatectomy [4].

Downregulation of CD44s (standard isoform), a transmembranous glycoprotein involved in cell-cell and cellmatrix interaction, has been reported to correlate with a high tumour grade (Gleason score $\geq 7$ ) in prostate cancer [5]. To our knowledge, most of the studies focusing on the prognostic implications of CD44s in prostate cancer investigated 'classical' acinar adenocarcinoma which most likely arose from the peripheral aspect of the gland. The prognostic implications of CD44s in IPC, which most commonly represents transition zone cancer, have not been reported yet.

Since radical prostatectomy (RP) is only rarely performed after detection of IPC in TURP specimens, we decided to collect cases from several institutions in the region of Berlin and Brandenburg. With this material, we are able to present the first comprehensive multicentre study which investigates immunohistochemically the largest study cohort of IPC patients to date. The focus was placed on the prognostic evaluation of the association between CD44s expression in IPC and conventional histopathologic outcome parameters (Gleason scores and histologic staging) performed on the subsequent RPs in a study cohort of 54 patients.

\section{Materials and Methods}

\section{Selection of Tumour Samples}

Retrospective computerized database analysis was performed in order to identify all patients with newly diagnosed, clinically unapparent IPC (neither palpable nor visible by imaging using transrectal 7-MHz ultrasonography) based on the histopathologic examination of TURP specimens resected due to symptomatic prostatic hypertrophy. In all cases, all the resected tissue was subjected to histopathologic examination in order to enable proper tumour staging, which was straightforward in all cases and based upon estimation of the extent of a tumour since equivocal cases (still T1a vs. just T1b) did not occur in our cohort. Subsequently, between 1999 and 2008, all patients underwent RP at the Departments of Urology affiliated to 2 hospitals in Berlin (Charité-University Medicine and Vivantes Clinic am Urban) and 5 hospitals in Brandenburg [HELIOS Clinic Bad Saarow, Clinic Hoyerswerda, Röhn Clinic Frankfurt (Oder), Clinic Bautzen and Ernst-vonBergmann Clinic Potsdam]. This analysis yielded a total of 54 patients (mean age: 65.9 years; range: $49-80$ years). None of the patients received radiotherapy, androgen deprivation, $5 \alpha$-reduc-
Table 1. Pertinent conventional clinicopathologic metrics of the study cohort

\begin{tabular}{ll}
\hline $\begin{array}{l}\text { Intervals of serum PSA } \\
\text { levels at the time of TURP }\end{array}$ & $\begin{array}{l}\text { Absolute (relative) } \\
\text { number of cases, } \mathrm{n}(\%)\end{array}$ \\
\hline Serum PSA levels & \\
$\leq 4.0 \mathrm{ng} / \mathrm{ml}$ & $24(47)$ \\
$4.1-10 \mathrm{ng} / \mathrm{ml}$ & $13(25.5)$ \\
$>10 \mathrm{ng} / \mathrm{ml}$ & $14(27.5)$ \\
Histopathologic staging and Gleason scores performed on \\
TURP chips \\
T1a/1b & $27(50) / 27(50)$ \\
Gleason sum $4-6$ & $51(94.4)$ \\
Gleason sum 7 & $3(5.6)$ \\
Gleason sum $8-10$ & $0(0)$ \\
Histopathologic staging and Gleason scores performed on \\
RP specimens \\
Gleason sum $4-6$ & $45(83.3)$ \\
Gleason sum 7 & $5(9.3)$ \\
Gleason sum $8-10$ & $4(7.4)$ \\
pT2 & $48(88.9)$ \\
pT3a/3b & $2(3.7) / 4(7.5)$ \\
pN0/pN1 & $54(100) / 0(0)$ \\
pR0/pR1 & $54(100) / 0(0)$ \\
\hline
\end{tabular}

Serum PSA levels were unavailable in 3 patients.

$\mathrm{pT}=$ Histopathologic tumour stage; $\mathrm{pN}=$ histopathologic nodal status; $\mathrm{pR}$ = histopathologic surgical margin status.

tase inhibitor (i.e. finasteride) therapy, repeated TURP or needle biopsy prior to radical surgery. The following data were recorded: serum prostate-specific antigen (PSA) levels at the time of TURP (unavailable in 3 patients), histopathologic staging (performed on TURP chips and on their subsequent RPs) and Gleason scores (performed on RPs) as well as surgical margin and nodal status. In RPs harbouring multifocal cancer, the composition of the dominant tumour nodule was representative of the entire tumour. Since Gleason scores have initially been assigned to only 17 of the TURP chips evaluated, the remaining cases were subsequently graded on their corresponding tissue microarray (TMA) spots.

Because most of the archived wax-embedded tissue blocks from the corresponding RPs have been discarded, a total of 12 (22.2\%) wax-embedded archived radicals could be retrieved for comparative CD44s immunostaining. The pertinent conventional clinicopathologic metrics of the study cohort are summarized in table 1 .

Central Review of Histopathology and TMA Construction

Haematoxylin and eosin (HE)-stained TURP chips were reviewed by 2 independent clinical pathologists (S.G. and A.E.) in order to select 1 representative section from each patient to be used for TMA construction and so account for the standardization of immunostaining procedures. During this review, special attention was paid to the presence and distribution of electrocau- 

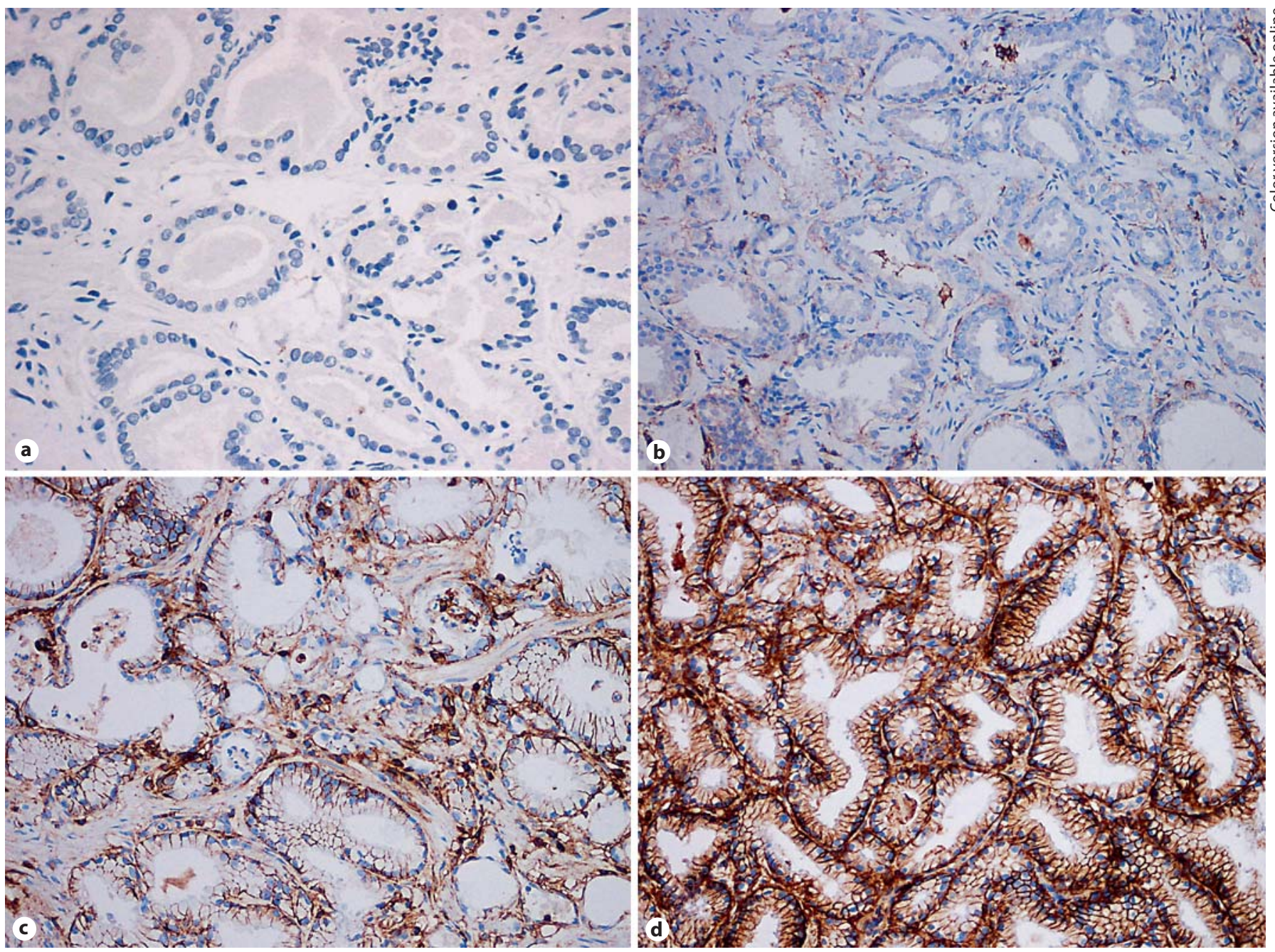

Fig. 1. IPC with a negative, b weak (1+), c moderate (2+) and d strong (3+) CD44s expression. Anti-CD44s. $\times 200$.

tery artefacts which were found to predominantly affect the fibromuscular stroma of the TURP chips while being negligible in their tumour-bearing areas (fig. 1). Prostatic intraepithelial neoplasia was not present in any of the specimens investigated.

Then, the corresponding paraffin-wax-embedded tissue blocks were retrieved from the pathology archives involved. Tumour-bearing areas for tissue retrieval were marked on HEstained sections, punched out of the paraffin block $(1.5 \mathrm{~mm}$ punch diameter) and inserted into a recipient block as previously described [6]. Due to tissue loss during arraying and sectioning, a total of 43 samples (79.6\%) remained available for immunostaining of CD44s. Serum PSA levels at the time of TURP were available in all of these 43 patients.

\section{Immunohistochemistry}

Freshly cut 4- $\mu \mathrm{m}$-thick TMA sections were used for immunohistochemistry. Of each of the 12 wax-embedded RP specimens retrieved, 1 representative tumour-bearing section from the tran- sition zone between the gland and the neighbouring areas of its peripheral zone was also subjected to immunohistochemistry. Following deparaffinization in graded alcohols and xylene, antigen demasking was achieved by heat retrieval $\left(100^{\circ} \mathrm{C}\right)$ in $0.01 \mathrm{M}$ citrate buffer for $30 \mathrm{~min}$, automatically performed by a Bond ${ }^{\mathrm{TM}}$ system (Vision Biosystems, Australia). Then, the primary antibody directed against CD44s [Lab Vision Neomarkers (monoclonal), clone 156-3C11; 1:100 dilution] was employed and incubated at $25^{\circ} \mathrm{C}$ for $1 \mathrm{~h}$. Subsequently, the section was washed with PBS and incubated with rabbit anti-mouse IgG 1:50, and following that, with mouse peroxidase-antiperoxidase conjugate 1:200. The enzymatic reaction was developed in a freshly prepared solution of diaminobenzidine ( $0.5 \mathrm{mg} / \mathrm{ml}$; Sigma, Deisenhofen, Germany) and $0.01 \%$ hydrogen peroxide in water.

Negative controls were prepared by omitting the primary antibody. Sections from an RP specimen not belonging to the study cohort that contained acinar adenocarcinoma as well as benign prostatic glands served as positive controls. 
The immunostained TMA section was evaluated by 2 independent clinical pathologists (S.G. and A.E.) blinded with respect to the specimens as well as to the staining grade previously assigned by the other pathologist. Then, an evaluation of the $12 \mathrm{im}$ munostained RP sections was performed a few weeks later without knowledge of the patients' identities or the CD44s staining grade previously assigned to their corresponding TMA spots. Briefly, the evaluation of marker expression was performed at $\times 200$ magnification. Positive staining was defined as having $>5 \%$ tumour cells showing positive membrane-associated staining with the staining intensity being graded as negative (0), weak $(1+)$, moderate $(2+)$ or strong (3+). Various examples of CD44s expression in IPC in the study cohort are illustrated in figure 1.

\section{Statistical Analyses}

Contingency tables and $\chi^{2}$ tests have been calculated in order to evaluate the association of dichotomized CD44s expression with other parameters.

The Spearman correlation was used to determine the magnitude and direction of the association between parameters. The non-parametric Wilcoxon test was employed in order to evaluate differences between the Gleason scores performed on TURP and RP specimens, respectively. Additionally, a multivariate regression model, with the Gleason scores performed on RP specimens as the target variable, was calculated in order to comparatively evaluate the impact of the different variables under study. To this aim, Gleason scores performed on RPs were dichotomized (Gleason score $<7$ vs. $\geq 7$ ). Cohen's weighted $\kappa$ statistics were used to assess inter-observer agreement concerning the staining evaluation. Briefly, $\kappa$ statistics are a measure of overall agreement without requiring assumptions concerning the 'correct' grade of marker expression. The value of $\kappa$ ranges from -1.0 to +1.0 . A value of 0 indicates chance agreement only, while a value of +1.0 indicates perfect agreement. A negative value denotes systematic disagreement between observers. It is generally accepted that a value of $0.001-0.20$ indicates slight agreement, $0.201-0.40$ fair agreement, $0.401-0.60$ moderate agreement, $0.601-0.80$ substantial agreement and 0.801-0.99 excellent agreement.

$\mathrm{p}<0.05$ was considered statistically significant. All calculations were performed using the statistical software package SPSS version 13.0.

\section{Results}

\section{Correlation between Conventional Clinicopathologic} Parameters

Histopathologically, all tumours investigated were found to be acinar adenocarcinomas. Ductal adenocarcinomas or other variants of prostate cancer were not investigated in our cohort.

Histologic staging performed on TURP chips (Tla and T1b) failed to correlate with Gleason scores $(\rho=0.08$; $\mathrm{p}=0.6)$ and with histologic staging $(\rho=0.03 ; \mathrm{p}=0.8)$ performed on their corresponding RPs.

Gleason scores assigned either to TURP chips or TMA spots failed to correlate with histologic staging performed on the TURP chips $(\rho=0.072 ; p=0.61)$ and on their corresponding RPs $(\rho=0.197 ; p=0.154)$ as well as with the patients' age $(\rho=0.19 ; p=0.168)$ and with serum PSA levels at the time of TURP $(\rho=0.165 ; p=0.247)$.

Gleason scores performed on TURP chips or TMA spots (mean: 5.26; range: 3-7) differed significantly from the Gleason scores assigned to their corresponding RP specimens (mean: 5.48; range: $3-9 ; \mathrm{p}=0.016$ ).

In comparison with the Gleason scores assigned to TURP chips or TMA spots, a total of 16 cases $(29.6 \%)$ required downgrading on their corresponding RP specimens while the remaining 38 cases $(70.4 \%)$ were found to have identical Gleason patterns on their subsequent RPs.

The patients' age at the time of prostatectomy failed to correlate with Gleason scores $(\rho=0.25 ; p=0.07)$ and with histologic staging $(\rho=0.03 ; p=0.08)$ performed on the corresponding RPs. However, there was a trend towards increased patient age in advanced cancer on RP in our study cohort (mean age in organ-confined disease 64 years vs. 67.4 years in organ-exceeding cancer).

Serum PSA levels at the time of TURP showed a significant correlation with Gleason scores $(\rho=0.63, \mathrm{p}=$ $0.01)$ and with histologic staging $(\rho=0.34, p=0.02)$ performed on the TURP chips but failed to correlate with Gleason scores $(\rho=0.19, p=0.17)$ and with histologic staging ( $\rho=0.23, p=0.12)$ performed on their corresponding RPs.

\section{CD44s Expression in the Tumour Samples}

Variable membrane-associated CD44s staining was observed in 39 (90.7\%) of the 43 IPCs immunohistochemically investigated (table 2). Staining results are summarized in tables 2 and 3. Notably, all of the 12 archived, wax-embedded RP specimens retrieved were assigned the same CD44s staining grades as previously allocated to TMA spots obtained from their corresponding TURP chips. All of the 12 RPs investigated showed homogeneous CD44s expression throughout the entire tumour tissue in terms of distribution and staining intensity (fig. 1).

According to the $\chi^{2}$ tests analysing dichotomized CD44s expression in the TURP chips, there were significant differences concerning the Gleason scores performed on TURP chips $(\mathrm{p}=0.009)$ and on RP specimens $(\mathrm{p}=0.006)$ as well as concerning histologic staging $(\mathrm{p}=$ 0.016 ) performed on RP specimens. In contrast, there were no significant differences concerning histologic staging performed on TURP chips $(\mathrm{p}=0.329)$, serum PSA levels $(\mathrm{p}=0.542)$ and the patients' age $(\mathrm{p}=0.325)$. 
Table 2. CD44s expression recorded in the TURP chips $(n=43)$ in relation to histopathologic tumour staging performed on TURP chips (T1a and T1b) and on their corresponding radical prostatectomies (pT2a to $\mathrm{pT} 3 \mathrm{~b}$ )

\begin{tabular}{lcrrrrrr}
\hline $\begin{array}{l}\text { Grade of } \\
\text { CD44s } \\
\text { staining }\end{array}$ & T1a & T1b & pT2a & pT2b & pT2c & pT3a & pT3b \\
\hline 0 & 4 & 0 & 2 & 0 & 0 & 1 & 1 \\
1 & 2 & 4 & 1 & 3 & 1 & 0 & 1 \\
2 & 9 & 8 & 11 & 1 & 5 & 0 & 0 \\
3 & 5 & 11 & 8 & 0 & 6 & 0 & 2 \\
\hline
\end{tabular}

pT = Histopathologic tumour stage.

Table 3. CD44s expression recorded in the TURP chips $(n=43)$ in relation to the Gleason scores assigned to their corresponding RPs

\begin{tabular}{llll}
\hline $\begin{array}{l}\text { Grade of CD44s } \\
\text { staining }\end{array}$ & $\begin{array}{l}\text { Gleason } \\
\text { scores 4-6 }\end{array}$ & $\begin{array}{l}\text { Gleason } \\
\text { score 7 }\end{array}$ & $\begin{array}{l}\text { Gleason } \\
\text { scores 8-10 }\end{array}$ \\
\hline 0 & 2 & 1 & 1 \\
1 & 2 & 1 & 3 \\
2 & 16 & 1 & 0 \\
3 & 14 & 2 & 0 \\
\hline
\end{tabular}

CD44s expression showed a significant correlation with the Gleason scores performed on the corresponding RP specimens (table 3; $\rho=0.905, \mathrm{p}<0.001$ ).

Forming subgroups, predicting Gleason scores $\geq 7$ on $\mathrm{RP}$ specimens by means of a low CD44s expression in the TURP chips was found to be feasible based upon a level of significance of $8 \%(\mathrm{p}=0.08)$, but failed to be significant based upon a level of significance set at $5 \%(\mathrm{p}=0.05)$.

Regarding the CD44 reactions of the 16 cases which were downgraded on the RP specimen compared with their corresponding TURP chips, the event of 'downgrading' failed to show any statistically significant association with CD44s expression.

The results obtained by means of a multivariate regression model in order to assess by comparison the impact of the different parameters on the dichotomized Gleason scores performed on RP specimens are summarized in table 4.

Concerning staining evaluation, there was substantial inter-observer agreement between both clinical pathologists in $88.6 \%$ of the immunohistochemically investigated cases $(\kappa=0.53 ; \mathrm{p}<0.001)$.
Table 4. Multivariate regression analysis to compare different variables in terms of their association with dichotomized Gleason scores ( $<7$ vs. $\geq 7$ ) performed on the corresponding RP specimens

\begin{tabular}{lllll}
\hline Variables & SE & HR & $95 \%$ CI & $\begin{array}{l}\mathrm{p} \\
\text { value }\end{array}$ \\
\hline Age (cont.) & 0.14 & 1.02 & $0.78-1.33$ & 0.908 \\
PSA (cont.) & 0.10 & 1.13 & $0.94-1.37$ & 0.201 \\
Gleason score on TURP (cont.) & 1.11 & 5.79 & $0.65-51.63$ & 0.115 \\
Tumour stage on TURP (cont.) & 1.46 & 0.42 & $0.02-7.27$ & 0.547 \\
CD44s (dichotomized) & 1.21 & 0.12 & $0.01-1.28$ & 0.080 \\
\hline
\end{tabular}

$\mathrm{SE}=$ Standard error; $\mathrm{HR}=$ hazard ratio; cont. = continuously

\section{Discussion}

Paul et al. [7] reported that IPC detected by TURP chips has an outcome similar to that detected by needle biopsy and recommended RP to be performed in selected candidates. However, clinical management of individual IPC remains challenging due to the unpredictability of its speed of progression.

Directing efforts at predicting the outcome of individual IPC prior to radical surgery, we aimed to assess the prognostic role of selected immunohistochemically detectable markers. According to our data, expression of prostatic acid phosphatase in TURP specimens is predictive of the histopathologic tumour stage in subsequent radical prostatectomies [8]. In the present study, we aimed to extend the currently limited panel of markers useful for prognostic evaluation in IPC.

Looking at our study cohort, 2 notions merit discussion. Firstly, approximately $37 \%$ of the clinically unapparent tumours were found to be at least stage pT $2 \mathrm{c}$ on RP. This figure might be explained by the frequent multicentricity of IPC. Secondly, approximately $17 \%$ of the IPCs were assigned Gleason scores $\geq 7$ on their corresponding RP in our cohort. Although a definite localization of the zonal origin of cancer is felt to be inappropriate in our cohort, these tumours might have arisen from the peripheral zone of the gland with subsequent intraglandular spread towards its transition zone.

We found complete concordance between the 12 waxembedded RPs retrieved and their corresponding TMA spots in terms of the CD44s staining grade assigned. Together with the observation that CD44s expression was found to be homogenous throughout the entire tumour tissue in the 12 RPs retrieved in terms of staining distri- 
bution and intensity (fig. 1), this finding supports the notion that the staining grades assigned to TMA spots represent the 'real' CD44s expression and are not substantially influenced by sampling errors.

Many cancer-associated genes such as CD44 are alternatively spliced [9]. Certain CD44 variants have been reported to have functional significance in prostate cancer [10]. Amongst these, the standard isoform CD44s has been extensively studied and its downregulation has been linked to high Gleason scores $[5,11,12]$. Therefore, owing to the well-known disagreement between the Gleason scores on TURP and on prostatectomy, CD44s was chosen to be investigated in our study which is the first to systematically assess the prognostic role of CD44 standard isoform in IPC [4]. We found a strong association between CD44s expression in the TURP chips and Gleason scores performed on their corresponding RPs which, according to multivariate regression analysis, was found to have the strongest predictive impact when dichotomized Gleason scores performed on RP specimens were compared with other prognostic variables. We feel the lacking significance of this important observation most likely to be attributable to the limited number of specimens immunohistochemically assessed. The same holds true in terms of forming subgroups according to CD44s expression which, according to our data, was found to be feasible at a level of significance of $8 \%$. In view of these statistical trends, CD44s expression might be helpful in discussing the clinical management of particular cases on a tumour board.

Concerning the association between CD44s expression and Gleason scores, our data are in keeping with the results previously reported by others, although, to our knowledge, these studies investigated 'classical' prostatic adenocarcinoma and did not focus on IPC which most commonly represents transition zone cancer $[5,11,12]$.

Recently, Aaltomaa et al. [13] reported downregulation of CD44s to be related to high tumour stage in prostate cancer. We failed to establish the latter correlation in our cohort, which might be attributable to the limited number of cases immunohistochemically assessed in our study.

The mechanisms behind our observations remain a matter of speculation. The bioactive signalling transmitter CD44s functions as a hyaluronan receptor involved in cell-cell and cell-matrix interaction [14]. Since the Gleason patterns histomorphologically reflect tumour architecture which is strongly influenced by the latter interactions, downregulation of CD44s expression might give rise to architectural disorganization, and this might be accompanied morphologically by a change in the Gleason score of the tumour.

Recently, adverse prognostic outcome in IPC has been linked to high immunoreactivity of $\mathrm{Ki}-67$ and neuroendocrine staining $[15,16]$. Therefore, CD44s might extend the currently limited panel of immunohistochemically detectable markers established to better define the natural course of individual IPC. P53 - which has been reported to predict resistance to radiotherapy or systemic treatment in prostate cancer - as well as other alternatively spliced CD44 isoforms might be further prognostically relevant targets to be addressed in future studies [17].

Since postsurgical serum PSA values depend upon a great body of different parameters, the possible association between CD44s expression and progression-free survival was not assessed in our investigation and invites larger prospective clinical studies.

In conclusion, because of the frequently encountered disagreement between the Gleason scores on TURP and on prostatectomy, CD44s might become a valuable adjunct to predict outcome in IPC prior to prostatectomy. The substantial reproducibility of its staining evaluation supports this concept. However, advanced prospective studies are clearly needed to define cut-point values of marker expression for separating aggressive tumours from their indolent counterparts, and to clarify possible associations between CD44s expression and clinical follow-up data (e.g. progression-free survival).

\section{Acknowledgements}

The authors gratefully thank Frank Dietrich, MD, Conrad Flössel, MD, Jan Jander, MD, Helmut Knispel, MD, Hartmut Lobeck, MD, Volker Loy, MD, Roland Pauli, MD, Volkmar Rosenthal, MD, and Wilko Weichert, MD, for their contribution to this work. We also gratefully thank the HELIOS Research Center for supporting the present study.

References

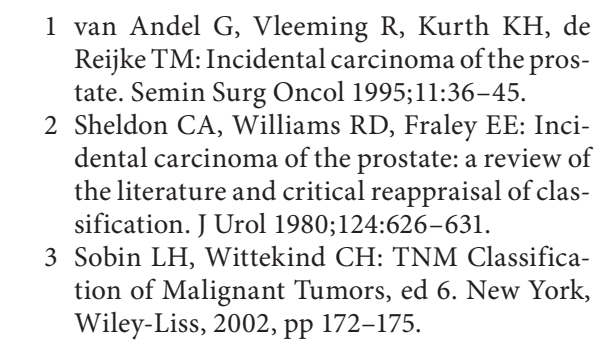

Pathobiology 2009;76:286-292 
4 Melchior S, Hadaschik B, Thüroff S, Thomas C, Gillitzer R, Thüroff J: Outcome of radical prostatectomy for incidental carcinoma of the prostate. BJU Int 2009;103:1478-1481.

5 Kallakury BV, Yang F, Figge J, Smith KE, Kausik SJ, Tacy NJ, Fisher HA, Kaufman R, Figge H, Ross JS: Decreased levels of CD44 protein and mRNA in prostate carcinoma: correlation with tumor grade and ploidy. Cancer 1996;78:1461-1469.

6 Kononen J, Bubendorf L, Kallioniemi A, Bärlund M, Schraml P, Leighton S, Torhorst J, Mihatsch MJ, Sauter G, Kallioniemi OP: Tissue microarrays for high-throughput molecular profiling of tumor specimens. Nat Med 1998;4:844-847.

7 Paul R, Knebel C, van Radenborgh H, Kübler H, Alschibaja M, Günther M, Hartung R: Incidental carcinoma of the prostate: can we and should we recommend radical prostatectomy? (in German). Urologe A 2005;44: 1054-1058.

$>8$ Gunia S, Koch S, May M, Dietel M, Erbersdobler A: Expression of prostatic acid phosphatase (PSAP) in transurethral resection specimens of the prostate is predictive of histopathologic tumor stage in subsequent radical prostatectomies. Virchows Arch 2009; 454:573-579.
9 Caballero OL, de Souza SJ, Brentani RR, Simpson AJ: Alternative spliced transcripts as cancer markers. Dis Markers 2001;17:6775.

10 Omara-Opyene AL, Qiu J, Shah GV, Iczkowski KA: Prostate cancer invasion is influenced more by expression of a CD44 isoform including variant 9 than by Muc18. Lab Invest 2004;84:894-907.

11 Noordzij MA, van Steenbrugge GJ, Verkaik NS, Schröder FH, van der Kwast TH: The prognostic value of CD44 isoforms in prostate cancer patients treated by radical prostatectomy. Clin Cancer Res 1997;3:805-815.

12 Aaltomaa S, Lipponen P, Viitanen J, Kankkunen JP, Ala-Opas M, Kosma VM: Prognostic value of CD44 standard, variant isoforms 3 and 6 and $\alpha$-catenin expression in local prostate cancer treated by radical prostatectomy. Eur Urol 2000;38:555-562.
13 Aaltomaa S, Lipponen P, Ala-Opas M, Kosma VM: Expression and prognostic value of CD44 standard and variant v3 and v6 isoforms in prostate cancer. Eur Urol 2001;39: 138-144.

14 Ouhit A, Abd Elmageed ZY, Abdraboh ME, Lioe TF, Raj MH: In vivo evidence for the role of CD44s in promoting breast cancer metastasis to the liver. Am J Pathol 2007;171: 2033-2039.

15 Robinson D, Aus G, Bak J, Gorecki T, Herder A, Rosell J, Varenhorst E: Long-term followup of conservatively managed incidental carcinoma of the prostate: a multivariate analysis of prognostic factors. Scand J Urol Nephrol 2007;41:103-109.

16 Theodoropoulos VE, Tsigka A, Mihalopoulou A, Tsoukala V, Lazaris AC, Patsouris E, Ghikonti I: Evaluation of neuroendocrine staining and androgen receptor expression in incidental prostatic adenocarcinoma: prognostic implications. Urology 2005;66: 897-902.

>17 Fernández PL, Hernández L, Farré X, Campo E, Cardesa A: Alterations of cell cycleregulatory genes in prostate cancer. Pathobiology 2002;70:1-10. 